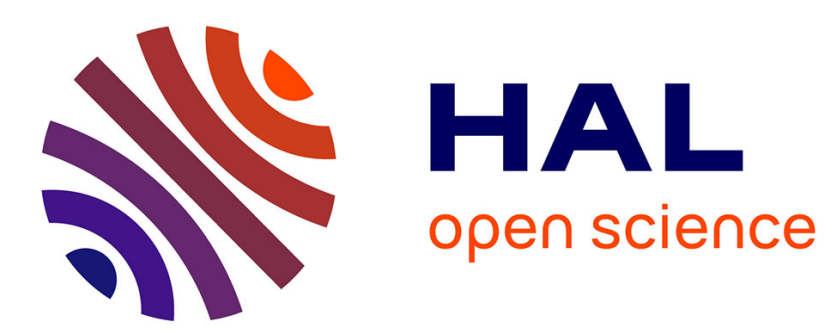

\title{
Pressure-induced reduction of the Landau-Peierls instabilities in a side-chain polymer liquid crystal with reentrant polymorphism
}

\author{
G Pépy, P Baroni, Laurence Noirez
}

\section{- To cite this version:}

G Pépy, P Baroni, Laurence Noirez. Pressure-induced reduction of the Landau-Peierls instabilities in a side-chain polymer liquid crystal with reentrant polymorphism. Physical Review E , 2003, 10.1103/PhysRevE.67.041714 . hal-01361934

\section{HAL Id: hal-01361934 \\ https://hal.science/hal-01361934}

Submitted on 7 Sep 2016

HAL is a multi-disciplinary open access archive for the deposit and dissemination of scientific research documents, whether they are published or not. The documents may come from teaching and research institutions in France or abroad, or from public or private research centers.
L'archive ouverte pluridisciplinaire HAL, est destinée au dépôt et à la diffusion de documents scientifiques de niveau recherche, publiés ou non, émanant des établissements d'enseignement et de recherche français ou étrangers, des laboratoires publics ou privés. 


\title{
Pressure-induced reduction of the Landau-Peierls instabilities in a side-chain polymer liquid crystal with reentrant polymorphism
}

\author{
G. Pépy, P. Baroni, and L. Noirez* \\ Laboratoire Léon Brillouin (CEA-CNRS), CE Saclay, 91191 Gif-sur-Yvette Cedex, France
}

(Received 16 October, 2002; published 29 April 2003)

\begin{abstract}
Cyanobiphenyl mesogens are known to exhibit partially bilayered smectic $A\left(S_{A d}\right)$ and also reentrant nematic $\left(N_{\text {re }}\right)$ phases. Nematic and smectic orders are coupled parameters which depend both on temperature and pressure. We report the first structural study of the influence of a hydrostatic pressure on the smectic phase. This study was carried out on a side-chain liquid crystalline polymer, by neutron diffraction using two specifically designed pressure cells. These results concluded first that the pressure acts on the phase elastic constants via a reduction of the layer fluctuations giving rise to a hardening of the phase together with an extension of the smectic domain towards higher temperatures. Second, the $S_{A d}-N_{\text {re }}$ phase transition temperature remains unchanged in the studied pressure range revealing that the polymer component plays an important role which allows us to subtract the associated packing interactions from the pressure-induced volume reduction.
\end{abstract}

DOI: 10.1103/PhysRevE.67.041714

PACS number(s): 61.30.-v, 64.70.Md, 61.25.Hq

\section{INTRODUCTION}

Liquid crystal phases are characterized by a long-range orientational order. Numerous studies on liquid crystals under hydrostatic pressure [1], display interesting pressureinduced phenomena which, indirectly, seem to indicate that the long-range order is disturbed. It has been shown, for example, that reentrant nematic phases can appear and that phase transition temperature shifts are commonly observed $[1,2]$. These pressure-induced effects are inferred from the packing properties of the system when the molar volume is reduced. However, despite the numerous investigations, none consider the eventual structural modifications which accompany the pressure-induced liquid crystallinity. We propose to describe the system in terms of pressure-induced correlation lengths and layer spacing which are relevant parameters to account for the phase structure. This structural investigation is particularly noteworthy in the smectic phase since it will be shown that the characteristic lengths can be tremendously modified under pressure underlining a correlated change of dynamics. This study is also unique in the case of side-chain liquid crystal polymers (SCLCPs). In SCLCPs, the liquid crystal ordering influences the chain conformation: it acts as a stress competing with the main chain entropy. Wang and Warner (WW) [3] proposed a reference model which foresees a wide variety of mesophases. Experimentally, most of SCLCPs display only a narrow succession of phases and conformations which correspond mainly to conventional nematic and smectic phases and to prolate and oblate mainchain conformations, respectively [4]. While chemists can tailor materials with discrete changes only (modifying for instance the spacer length), the pressure is a continuous variable; the application of a hydrostatic pressure introduces a

\footnotetext{
*Corresponding author. Email address: noirez@1lb.saclay.cea.fr
}

specific volume variation which is, by way of the liquid crystalline phase, anisotropically distributed.

From a theoretical point of view, it is useful to point out that the specific volume is taken into account into the models. The analytical theory proposed by WW for nematic sidechain liquid crystal polymers is based on five interactions between the SCLCP constitutive components, and the occupancy ratio for the side-chain mesogens to the volume occupied per monomer. Many variants of the three main nematic structures (called $N_{\mathrm{I}}, N_{\mathrm{II}}$, and $N_{\mathrm{III}}$ and corresponding to the oblate and two types of prolate main chain conformations, respectively) are predicted, with either strong or poor ordering of the main chain. Finally, based on molecular calculations, Dowell [5] foresees modified smectic structures by only slightly changing the SCLCP's molecular parameters.

Pressure is thus a very convenient tool to test the stability of SCLCPs, to compare the behavior of their highly viscous phases to those of low-molecular-weight liquid crystals [2], to explore its influence on the coupling with the polymer main-chain conformation, and maybe to reveal an effect of the glass transition due to an exacerbated polymeric nature of the SCLCP under pressure [6].

This paper reports thus the first structural information, obtained in situ, by applying a hydrostatic pressure on a SCLCP. Neutron diffraction was first carried out with a pressure cell reaching pressures up to 400 bar [7] and then extended, using an improved pressure setup, to a high pressure regime up to 1200 bar. The main parameters affected by a pressure variation will be identified and their evolution will be interpreted in terms of molecular interactions. In a second step, the results corresponding to a higher pressure range (up to 1200 bar) will be displayed. An overall analysis indicating the principal features of the pressure-induced behavior will conclude the paper.

The sample studied, noted PA-CN, is a polyacrylate main chain to which a mesogen consisting of a cyanobiphenyl group is linked: 


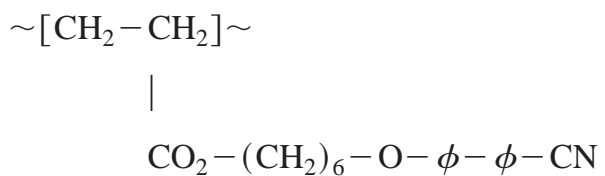

This polymer exhibits a reentrant behavior [8]. The sample studied here has a molecular weight of $M_{W}=98000$ and a polydispersity $I=12$. It displays the following phase sequence (DSC measurements $10^{\circ} \mathrm{C} / \mathrm{min}$ ):

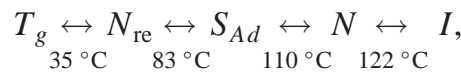

where $I$ indicates the isotropic phase, $N$ the high temperature nematic phase, $S_{A d}$ a partially bilayered smectic- $A$ phase, $N_{\text {re }}$ a low temperature (reentrant) nematic phase, and $T_{g}$ the glassy state. This peculiar behavior is attributed to a competition between different short range but strong electric dipolar interactions induced by the terminal cyano group which favors the formation of antiparallel molecular association (pairing) to the detriment of long-range monolayers (nonpolar-nonpolar interactions) [9]. Pairing produces smectic phases characterized by layer thicknesses not commensurate but longer than the molecular length (the $S_{A d}$ phase). The competition between different arrangements is temperature dependent, leading in specific cases to the restoration of the usual long-range monolayer phase [10]. The $N_{\mathrm{re}}-S_{A d}$ phase transition temperature corresponds thus to the point where these antagonist interactions are balanced.

\section{EXPERIMENT}

The polymer sample was encapsulated under vacuum in an internal container with a flexible wall made of Kapton (from Du Pont de Nemours). In the pressure cell, this container is surrounded by $\mathrm{D}_{2} \mathrm{O}$, the pressure transmitting medium. In a first step, the pressure study was carried out using a zircalloy cell [7] enabling us to reach a nominal pressure of 400 bar and a temperature of $150{ }^{\circ} \mathrm{C}$. In a second step, the experiment was extended up 1200 bars at $150{ }^{\circ} \mathrm{C}$ with a new pressure cell (Fig. 1). Its body is made of beryllium copper and the windows are made of sapphire, allowing a direct optical sample observation and the possibility to carry out small angle neutron scattering experiments to access to pressure-induced conformations of the polymer main chain [11].

The measurements were performed using the 3T1 diffractometer of the Laboratoire Léon Brillouin at the Orphée reactor, Saclay. It consists of characterizing by neutron diffraction the smectic structure associated with the 001 reflection at $q \approx 0.188 \AA^{-1}$. The neutron wavelength was $2.356 \AA$. During the whole experiment, the pressure cell was kept in the gap of a $1.4 \mathrm{~T}$ electromagnet in order to align the mesophase monodomains. The scattering angle was scanned over the position of the 001 smectic reflection, through the full temperature range, for a set of pressure values. The shape of the 001 smectic reflection measured with an angular resolution of $20^{\prime}$ was approximated to a Gaussian curve:

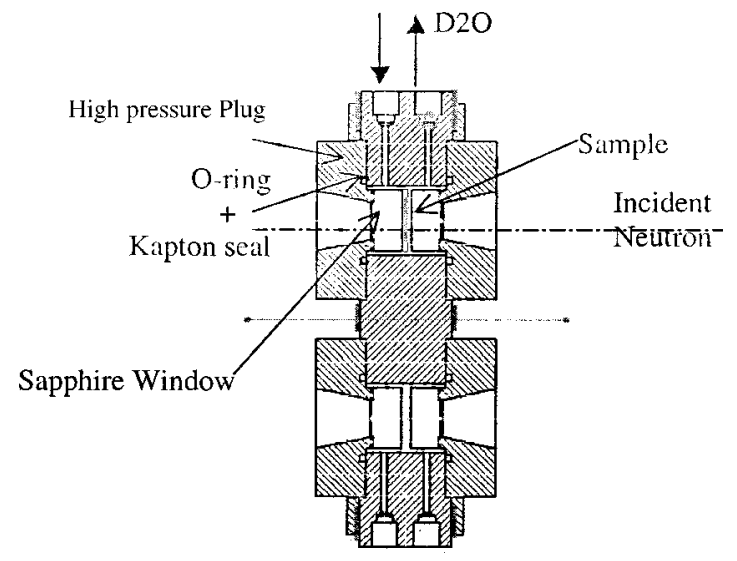

FIG. 1. Schematic representation of the high pressure cell (double cell). The high pressure plug exercises the pressure on the sapphire windows. The O-ring and the Kapton seal ensure the sealing of the whole set.

$$
S(|\vec{q}|)=S_{0} \exp \left\{-\left[\frac{16}{\ln 2^{2}}\left(\frac{\left(q_{h}-q_{0}\right)^{2}}{\Delta q_{\|}^{2}}+\frac{q_{\perp}^{2}}{\Delta q_{\perp}^{2}}\right)\right]\right\},
$$

where $S_{0}$ is the susceptibility corresponding to the intensity maximum at $\left(0,0, q_{0}\right)$. The peak position $q_{0}$, the intensity maximum $S_{0}$ (determined from a longitudinal scan), and the widths $\Delta q_{\|}, \Delta q_{\perp}$ along the longitudinal axis (parallel to the director) and along the transverse direction, respectively, are systematically determined versus temperature and pressure. The widths $\Delta q_{\|}, \Delta q_{\perp}$ are related to the respective correlation lengths $\xi_{\|}, \xi_{\perp}$ via $\xi=4 \pi / \Delta q$. The correlation lengths are corrected from the instrumental resolution broadening effects using the following approximate relationship between the measured width and the effective one: $\Delta q_{\text {measured }}^{2}$ $=\Delta q_{\text {resolution }}^{2}+\Delta q_{\text {effective }}^{2}$ (the Rietveld approximation). The longitudinal and the transverse resolution values, deduced from a single crystal scattering, were $0.028 \AA^{-1}$ [full width at half maximum (FWHM)] and $0.008 \AA^{-1}$ (FWHM), respectively, for the current spectrometer configuration.

\section{RESULTS AND DISCUSSION}

We shall present and analyze a first set of results obtained in the intermediate pressure range (from 1 to 400 bar). First and second sets of results differ by the use of two distinct pressure cells giving access to low and high pressure ranges, respectively. By increasing the pressure from 1 to 400 bar, several major modifications attributed to the pressure effect can be noticed.

(a) Figure 2 displays the evolution of the 001 intensity maximum $S_{0}$ versus temperature. These curves present similar shapes characterized by temperature intervals defined by a sharp intensity evolution (identified at atmospheric pressure as the emergence of the smectic phase). Whatever the pressure, its effect on $S_{0}$ becomes notable only above a critical temperature $T=83^{\circ} \mathrm{C}$ where the pressure-induced $S_{0}$ values depart clearly from the atmospheric values, reaching up to the sevenfold at 380 bar. Since the intensity is connected to the number of scatterers involved in the smectic 


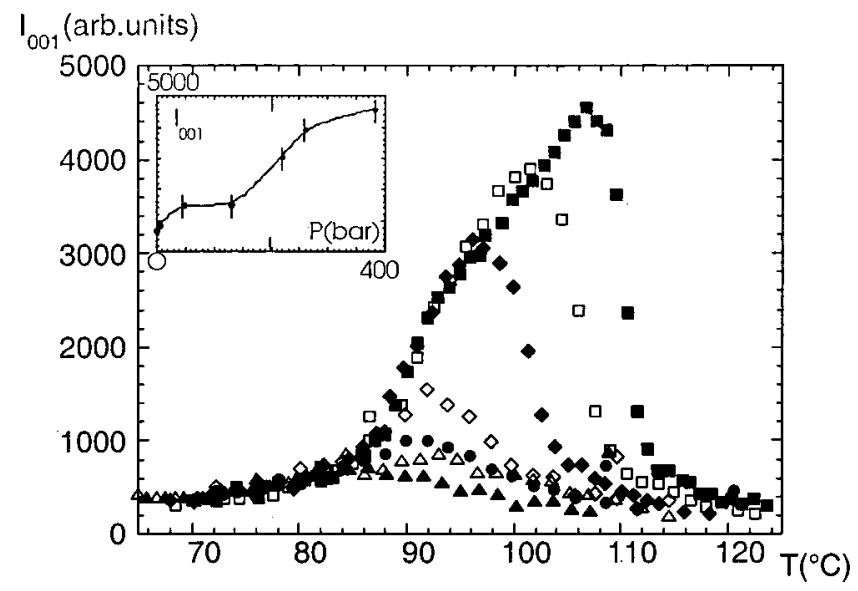

FIG. 2. Evolution of the 001 intensity (maximum value) scanned along the longitudinal axis (along the director) versus temperature, from atmospheric pressure up to 380 bar. The convergence of the intensity values at low temperature indicates that the $N_{\mathrm{re}}-S_{A d}$ remains unaffected by the external pressure. $\boldsymbol{\Lambda}, 1$ bar; $\triangle, 7$ bar; $\boldsymbol{Q}$, 50 bar; $\diamond, 130$ bar; $\diamond, 220$ bar; $\square, 260$ bar; $\mathbf{\square}, 380$ bar. In the inset, the evolution of the 001 intensity is displayed versus pressure; a leap in the intensity variation is observed at about 200 bar.

ordering, the $S_{0}$ increase indicates that the pressure induces an improvement of the localization of the mesogens into the layers; the dipolar pairing at the origin of the partially bilayered structure is reinforced.

(b) Figure 3(a) shows the correlation length $\xi_{\|}$along the director (longitudinal direction) as a function of the temperature at different pressure values. $\xi_{\|}$reaches values about 400 $\AA$ at $100^{\circ} \mathrm{C}$ at atmospheric pressure. Under pressure, the $\xi_{\|}$ curve is shifted, along the reentrant nematic branch, towards higher correlation values. Along the direction perpendicular to the director (transverse direction), the correlation length $\xi_{\perp}$ is also deeply affected by the pressure [Fig. 3(b)]. Values of $\xi_{\perp}$ of about $400 \AA$ are measured at atmospheric pressure which extends up to $2500 \AA$ at 380 bar. These correlation length evolutions indicate that the pressure reduces mainly the smectic layer distorsion; this is here illustrated by the huge decrease of the magnitude of the mesogen fluctuations which give rise to the broadening of the smectic reflection; the smectic layers formed under pressure present a much higher layer parallelism and the interlayer mesogen distance is repeated over a much larger range. Both elastic constants related to the layer dilatation and the undulations are reduced. These modifications are coherent with an increase of the intensity reflection (Fig. 2).

(c) Finally, the smectic layer distance itself is also pressure dependent (Fig. 4). The evolution is mainly observable above $83^{\circ} \mathrm{C}$. Below this temperature, no long-range smectic arrangement exists anymore and the short range correlation distance becomes relatively insensitive to the applied pressure. The examination of the position of the 001 reflection reveals that the layer spacing increases sensibly at moderate pressure with respect to the atmospheric pressure $(\approx 4 \%$ of layer spacing increase from 7 to $27 \mathrm{bar}$ ) whereas it seems to be stabilized from 27 bar up to 310 bar at a constant value $d \approx 34 \AA$. This layer distance increase versus pressure is of
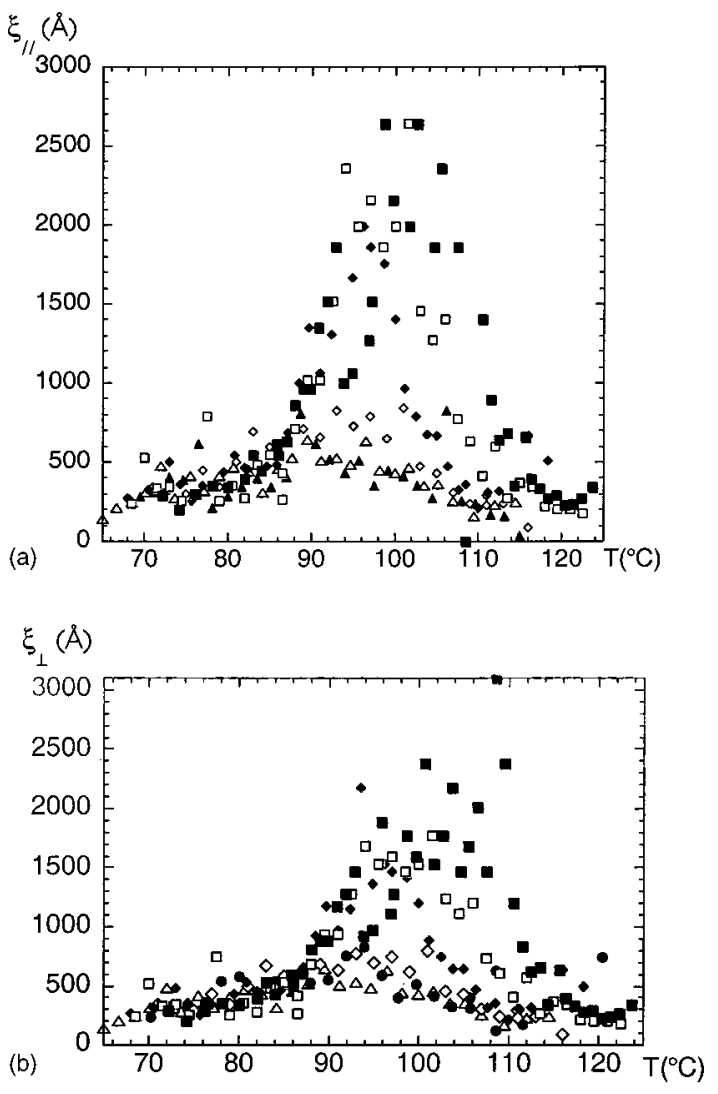

FIG. 3. (a) Evolution of the correlation length along the director $\xi_{\|}(\AA)$ (longitudinal axis). $\boldsymbol{\Delta}, 1$ bar; $\triangle, 7$ bar; $\diamond, 130$ bar; $\diamond, 220$ bar; $\square, 260$ bar; $\square, 380$ bar. (b) Evolution of the correlation length perpendicular to the director (transverse direction) $\xi_{\perp}(\AA)$ : $\triangle, 7$ bar; $\bullet, 50$ bar; $\diamond, 130$ bar; $\diamond, 220$ bar; $\square, 260$ bar; $\boldsymbol{\square}, 380$ bar. These values are deduced from the full widths at half maximum (FWHM) of the 001 smectic reflection. The values corresponding to the phase transition temperatures at atmospheric conditions are indicated by a dotted line.

the same order as the effect of a temperature decrease. It is consistent with the previous interpretations, i.e., the layer distance decrease is coherent with a reduction of the layer distorsion.

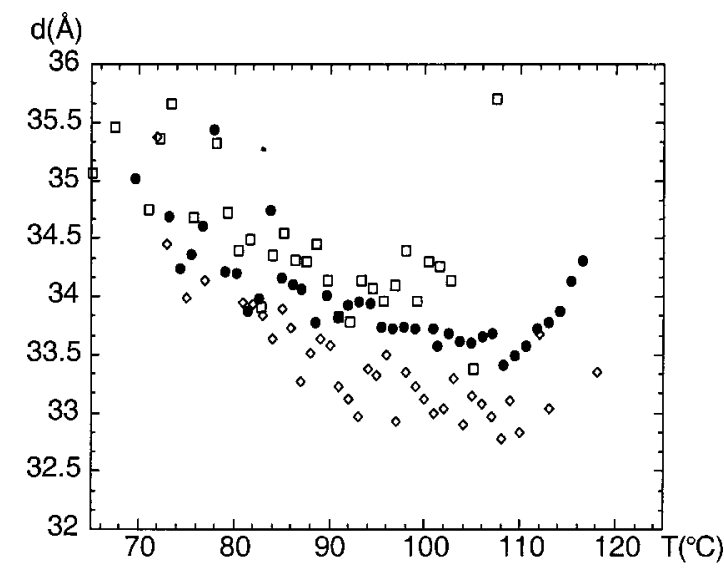

FIG. 4. Evolution of the smectic layer thickness versus temperature from 3 to 380 bar. $\diamond, 3$ bar; $\square, 27$ bar; $\bullet 380$ bar. 


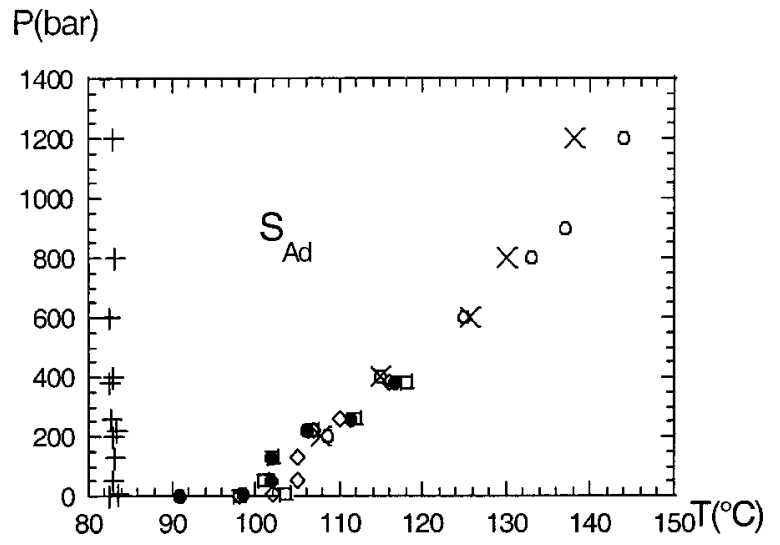

FIG. 5. Determination of the $S_{A d}$ temperature domain versus pressure. Variations of the $N_{\mathrm{Re}^{-}} S_{A d}(+)$ and $S_{A d}-N$ transition temperatures versus pressure. The values are deduced from both the correlation length variation $\xi_{\|}$at high pressure measurements $(\times)$ and low pressure measurements $(\mathbf{)})$, and from the intensity evolution at low pressure measurements $(\diamond)$ and high pressure measurements $(\bigcirc)$.

The three preceding points indicate that all the structural parameters of the smectic phase are modified under pressure. To determine the limits of the phase transitions, let us take as an internal reference the value taken by the correlation length $\xi$ at atmospheric pressure, corresponding to the $N_{\mathrm{re}}-S_{A d}$ and the $S_{A d^{-}} N$ transition temperatures deduced from DSC and microscopy measurements $[8(\mathrm{~b})]$. At both transitions $\left(T_{N_{\mathrm{re}} S_{A d}}\right.$ (atmospheric pressure $)=83{ }^{\circ} \mathrm{C}$ and $T_{S_{A d} N}($ atmospheric pressure $)=110^{\circ} \mathrm{C}$ ), the correlation lengths are approximately equal $\xi_{\perp \mathrm{atm}} \cong \xi_{\| \mathrm{atm}}=4 \pi / \Delta q=310 \pm 10 \AA$. Using the $\xi_{\| \text {atm }}$ and $\xi_{\perp \text { atm }}$ values as an internal criterium to define the limits of the smectic phase, one can estimate the high and low nematic-smectic transition temperatures $\left(T_{N_{\mathrm{re}} S_{A d}}\right.$ and $T_{S_{A d^{-}} N}$, respectively) and examine their displacement versus pressure. The following observations can be drawn with increasing pressure

(a) From the evolution of the correlation lengths $\xi_{\|}$and $\xi_{\perp}$ [Figs. 3(a) and 3(b)], it can be established that the $S_{A d^{-}} N$ transition is shifted towards higher temperatures. Both parallel and perpendicular correlation lengths give coherent values, indicating that the transition temperature follows approximately the same linear progression versus pressure: $\left(T_{S_{A d^{-} N^{-}}} 98\right)\left({ }^{\circ} \mathrm{C}\right) \cong 0.046 . p$ (bar) (Fig. 5). This pressure dependence is a simple variation which indicates that one parameter is sufficient to describe the transition. Neglecting the volume variation, such a pressure dependence means that it can be described by a simple variation of the extension (number) of fluctuations involved in the transition. The dependence and the slope $d T / d p \cong 4.6^{\circ} \mathrm{C} / \mathrm{kbar}$ are coherent with what is found for secondary transitions [6]. The comparison of Figs. 3 and 2 shows that the evolution of $\xi$ versus temperature and pressure is remarkably similar to the 001 intensity variation. At high pressure (380 bar), the $S_{A d^{-}} N$ transition coincides with the abrupt fall of correlation length $\xi$ (Fig. 3) which also corresponds to a leap of the 001 intensity (Fig. 2). For pressures below 380 bar, a progressive and simultaneous decrease of both parameters (intensity and cor- relation lengths) is observed by increasing the temperature before entering into the high temperature nematic phase. The correlation lengths and the values of the 001 intensity seem to be thus strongly coupled so that the 001 intensity value at $T_{S_{A d^{-N}}}$ (atmospheric pressure) can also be used as a criterium to determine the borders of the smectic phase. On Fig. 5 are superposed the transition temperatures deduced from both correlation lengths and the 001 intensity evolution criteria. The transition temperatures deduced from the different parameters are in good agreement. It is remarkable to notice that whatever the pressure, with increasing the temperature, all the parameters $\left(S_{0}, \xi_{\|}, \xi_{\perp}\right)$ keep on the same master branch up to their curve maximum (see in particular Fig. 2). The displacement of the 001 intensity maximum along this branch is clearly observed from 130 bar to 380 bar; the pressure essentially extends the asymptotic limit up to the highest value of the intensity. This evolution is the signature of a pressure-induced stabilization of the smectic fluctuations into a long range smectic order which, once formed, is no longer sensitive to a further pressure increase (within this studied pressure range), providing only a shift of the $S_{A d}-N$ transition temperature towards higher temperatures. These fluctuations are specific to a two-dimensional (2D) arrangement of the smectic state (also called Landau-Peierls instabilities); their destruction means that the smectic arrangement tends to a crystalline structure [12]. At atmospheric pressure, the thermal activation still allows short range smectic fluctuations and thus produces a retardation of the $N-S_{A d}$ transition temperature which is progressively shortened under pressure.

(b) On the pressure-temperature evolution diagrams of the longitudinal and the transverse correlation length $\xi_{\|}$and $\xi_{\perp}$, respectively, [Figs. 3(a) and 3(b)], and of the 001 intensity (Fig. 2), the $N_{\text {re }}-S_{A d}$ transition can be easily identified by the intersection point of the curves at different pressures which superpose at low temperature. This point corresponds to $T_{N_{\mathrm{re}} S_{A d}} \cong 83^{\circ} \mathrm{C}$, which is strictly the $N_{\mathrm{re}^{-}} S_{A d}$ transition temperature already determined at atmospheric pressure. None of the structural parameters of the $N_{\mathrm{re}}-S_{A d}$ transition is affected by the external pressure up to 400 bar; the $N_{\text {re }}-S_{A d}$ transition temperature remains invariant with pressure. This result is consistent with the previous observation which reveals the asymptotic development of a pressure-temperature branch (particularly observable on Fig. 2). Finally, the $N_{\text {re }}-S_{A d}$ transition temperature invariance is also compatible with the absence of the pressure-induced layer spacing change below $88^{\circ} \mathrm{C}$ (Fig. 4). The interactions involved are thus not, in this studied range, affected by the pressure.

In a second step, the present study is extended to pressures up to 1200 bar using another and new designed pressure cell equipped with sapphire windows (Fig. 1) [10]. Only three parameters [the 001 intensity maximum $S_{0}$ (Fig. 6) determined from the longitudinal scan, the full width at half maximum along the longitudinal axis $\Delta q_{\|}$giving rise to the correlation length $\xi_{\|}$(Fig. 7), and the peak position $q_{0}$ (Fig. 8)] are measured. Because of the similitude between the pressure induced evolutions of the correlation lengths and the intensity maximum, it will be supposed that the two measured parameters $\left(S_{0}\right.$ and $\xi_{\|}$) are sufficient to describe the pressure effects and more particularly the transition tem- 


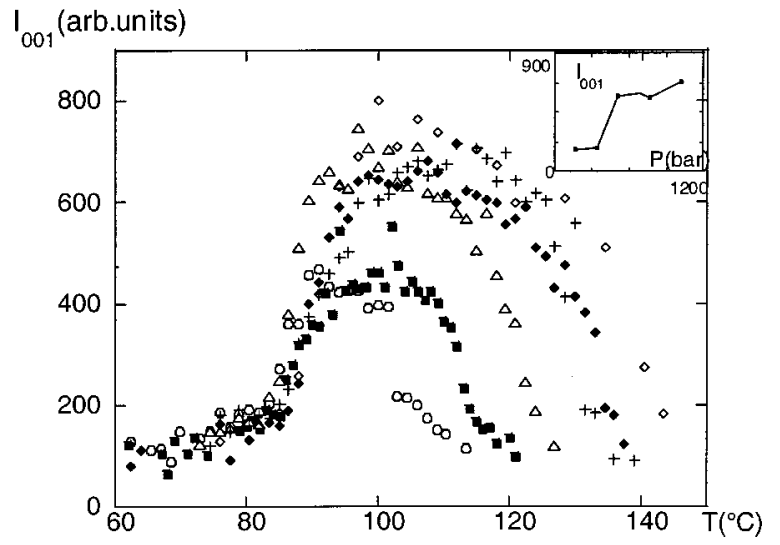

FIG. 6. High pressure evolution of the 001 intensity (maximum value) scanned along the longitudinal axis (along the director) versus temperature from 200 to 1200 bar. $\bigcirc, 200$ bar; $\square, 400$ bar; $\triangle$, 600 bar; +, 800 bar; $\diamond, 900$ bar; $\diamond, 1200$ bar. In the inset, the evolution of the 001 intensity is displayed versus pressure. The values tend to saturate above 600 bar.

peratures. The measurements are carried out from 200 bar to 1200 bar, thus covering partly the preceding lower pressure diagram. From 400 to 1200 bar, one can notice an apparent continuity of the behavior in agreement with the lower pressure evolution; the 001 intensity maximum reaches at 1200 bar almost twice the value displayed at 380 bar (Fig. 6). However, this progression is not linear; the pressure has a more pronounced effect on the intensity maximum above 300 bar and stationary values seem to be reached as the pressure approaches 600-1200 bar (Fig. 6 and inset).

The quasi-invariance of the intensity maximum above 600 bar up to $110^{\circ} \mathrm{C}$ would indicate that no smectic fluctuations are present anymore in the nematic phase which has been converted into a long range rigid smectic order. Since the pressure mainly acts on the layer distorsion, the existence of a characteristic pressure above which the layers rigidity is first clearly enhanced and then saturated, is related to a nonlinear modification of the layer elastic constants which describe the layer undulation dynamic [13,14]. In the Landau-de Gennes-McMillan description [12], the elastic constants appear as a coefficient of the development of the free energy variation. The elastic constant called $B$ expresses the restoring force for fluctuations (i.e., restoring the layer thickness). $\quad B$ is supposed to vary as $\xi_{\|}$. The apparent stationary values obtained above 600 bar would then indicate that a limit of elastic constants has been reached. It is opened to question to know what would be the issue of a further pressure increase on these parameters, and if it could make a new phase transition occur. Finally, the study in the high pressure range of the intensity maximum also confirms the two tendencies observed at lower pressure; the $N_{\mathrm{re}}-S_{A d}$ transition temperature still remains invariant under high pressure till 1200 bar, while the higher $S_{\mathrm{Ad}}-N$ transition temperature goes on increasing following approximately a linear variation with the pressure (Fig. 5).

\section{CONCLUSIONS}

This neutron diffraction study of this reentrant polymer liquid crystal has shown the relevance of the determination

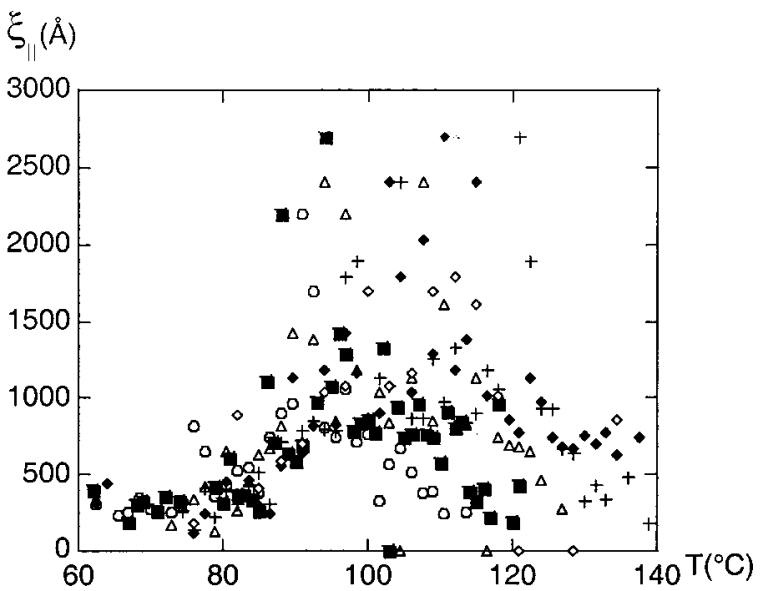

FIG. 7. High pressure behavior of the correlation length $\xi_{\|}$versus temperature at various pressure values from 200 to $1200 \mathrm{bar} . \bigcirc$, 200 bar; $\mathbf{\square}, 400$ bar; $\triangle, 600$ bar; +, 800 bar; $\diamond, 900$ bar; $\diamond, 1200$ bar.

of the structural phase parameters (correlation lengths) to characterize the pressure-induced modifications of the smectic phase. Concerning the $N-S_{A}$ transition temperatures, an extension of the smectic interval towards higher temperatures is observed, similarly to what is usually observed in low molecular weight liquid crystals in the low pressure regime $[1,2]$. The structural analysis has revealed in addition that the transition temperature shift is associated with a pressure-induced enhancement of the smectic ordering. Indeed, the significant increase of the correlation lengths (and the correlated intensity increase) with increasing pressure demonstrates a reinforcement of the smectic phase, whose phase domain extends only towards higher temperature and which corresponds to a reduction of the layer undulations (Landau-Peierls instabilities) and a reduction of the layer spacing (Figs. 4 and 8). The pressure acts thus at the scale of the elastic constants of the smectic layers. Quantitatively, the characteristic features observed below 400 bar still exist up to 1200 bar with a possible saturation of the maximum intensity, which might mean a quasisuppression of the smectic undulation instabilities.

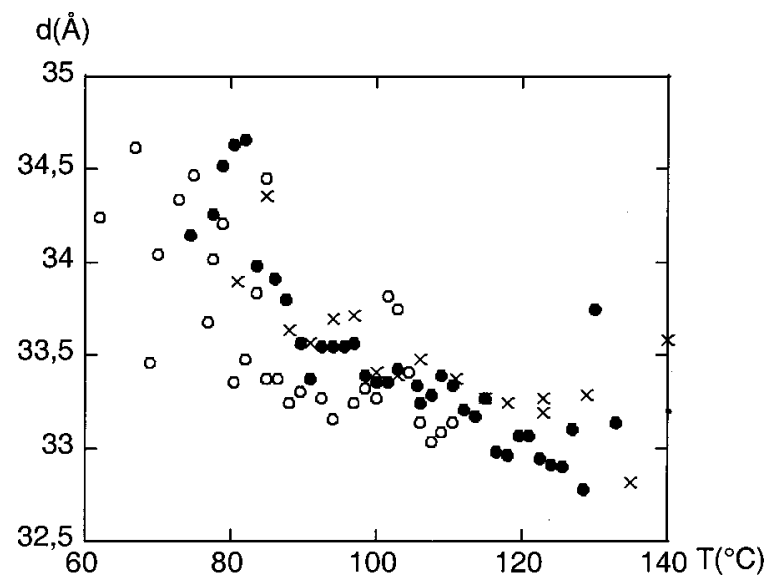

FIG. 8. Evolution of the smectic layer thickness versus temperature in the high pressure regime. $\bigcirc, 200$ bar; $\bullet, 800$ bar; $\times, 1200$ bar. 
Concerning the reentrant nematic phase, while pressure studies of small molecules of reentrant cyanobiphenyl liquid crystals have shown that the nematic-smectic separation line describes an ellipse [2(b)], the PA-CN pressure-temperature phase diagram does not reproduce this shape. The linear dependence of the high temperature $S_{A}-N$ transition temperature with the pressure upwards 1200 bar, is only consistent with the low pressure region of small liquid crystalline molecules. Contrary to small liquid crystalline molecules, the $N_{\text {re }}-S_{A d}$ transition temperature remains surprisingly unaffected by the pressure. Measurements carried on lowmolecular-weight liquid crystals have shown that the density is unchanged versus pressure at the $N_{\mathrm{re}}-S_{A d}$ transition due a full packing of the pairing interaction (and thus the $N_{\mathrm{re}}-S_{A d}$ transition temperature changes) [2(b)], revealing that the reentrant transition has a local origin and that, in the case of the polymer homologue, it is not submitted to the pressureinduced reduction of the density. This feature is coherent with the absence of variation of the smectic fluctuation layer thickness below $88^{\circ} \mathrm{C}$ (in the $N_{\text {re }}$ phase). We can first conclude that the pressure range applied here is not sufficiently large to affect locally the pairing interaction. Second, since the structure and the temperature of the $N_{\mathrm{re}}-S_{A d}$ transition remains unchanged by varying the pressure, it implies that the won energy has been converted in a nonliquid crystalline specific volume variation. The differences are between low molecular weight compounds and SCLCPs being inferred to the polymeric nature; the former compensates the whole pressure-induced volume variation and thus should be strongly affected by the pressure change. Finally, in the smectic phase, since polymer main chains are confined between the mesogen layers [15], and since pressure is able to suppress the layer distorsion and to condense the smectic fluctuations into a long range smectic order towards temperatures higher than at atmospheric pressure, it acts consequently on the polymer chain entropy. We have thus demonstrated that pressure exacerbates antagonist tendencies on SCLCPs giving rise to a specific pressure-dependent behavior. Future studies on the pressure-induced polymer conformation seems to be therefore particularly expected.

\section{ACKNOWLEDGMENT}

The authors are very grateful to G. Kirsch for the synthesis of the monomer and the polymerization of the sample.
[1] S. Chandrasekhar and R. Shashidhar, Adv. Liq. Cryst. 4, 83 (1979).

[2] (a) L. Liebert and W. B. Daniels, J. Phys. (Paris) 38, 333 (1977); A. N. Kalkura, R. Shashidhar, and N. Subbramanaya Rayj Urs, J. Phys. (France) 44, 51 (1983); (b) P. E. Cladis, D. Guillon, F. R. Bouchet, and P. L. Finn, Phys. Rev. A 23, 2594 (1981); P. E. Cladis, R. K. Bogardus, W. B. Daniels, and G. N. Taylor, Phys. Rev. Lett. 39, 720 (1977).

[3] X. J. Wang and M. Warner, J. Phys. A 19, 2215 (1986); M. Warner, Philos. Trans. R. Soc. London, Ser. A 348, 59 (1994).

[4] (a) Side Chain Liquid Crystal Polymers, edited by C. B. McArdle (Chapman and Hall, New York, 1989); (b) L. Noirez, P. Keller, and J. P. Cotton, Liq. Cryst. 18, 129 (1995).

[5] F. Dowell, Phys. Rev. A 28, 6 (1983); 28, 3520 (1983); F. Dowell, ibid. 28, 6 (1983); 28, 3526 (1983); Mol. Cryst. Liq. Cryst. 155, 457 (1988).

[6] R. W. Warfield and B. Hartmann, Polymer 23, 1835 (1982).

[7] G. Pépy, P. Baroni, and L. Noirez, Mol. Cryst. Liq. Cryst. Sci. Technol., Sect. A 364, 1235 (2001). Note: in this reference, the data corresponding to the transverse and longitudinal correla- tion lengths have been exchanged.

[8] (a) T. I. Gubina, S. G. Kostromin, R. V. Talroze, V. P. Shibaev, and N. A. Plate, Vysokomol. Soedin., Ser. B 28, 394 (1986); (b) P. Le Barny, J. C. Dubois, C. Friedrich, and C. Noël, Polym. Bull. (Berlin) 15, 341 (1986); (c) N. Lacoudre, A. Le Borgne, N. Spassky, J. P. Vairon, P. Le Barny, J. C. Dubois, S. Esselin, C. Friedrich, and C. Noël, Mol. Cryst. Liq. Cryst. 155, 113 (1988).

[9] J. O. Indekeu and A. W. Becker, Phys. Rev. A 33, 1158 (1986).

[10] F. Hardouin, G. Sigaud, M. F. Achard, and H. Gasparoux, Phys. Lett. 71A, 347 (1979).

[11] G. Pepy, P. Baroni (unpublished).

[12] P. G. de Gennes and J. Prost, The Physics of Liquid Crystals, 2nd ed. (Clarendon Press, Oxford, 1993).

[13] P. G. de Gennes, Mol. Cryst. Liq. Cryst. 21, 49 (1973); J. Phys. (Paris), Colloq. 30, C4-65 (1969).

[14] J. P. Poirier, Introduction to the Physics of the Earth's Interior (Cambridge University Press, Cambridge, England, 2000).

[15] L. Noirez, P. Keller, P. Davidson, F. Hardouin, and J. P. Cotton, J. Phys. (France) 49, 1993 (1988). 